Babak Jamshidi*, Shahriar Jamshidi Zargaran and Mansour Rezaei

\title{
Modelling spreading of an infection using time series by a novel family of models; fitting the time series of the confirmed cases of COVID-19 in China
}

https://doi.org/10.1515/em-2020-0013

Received May 31, 2020; accepted November 6, 2020; published online November 27, 2020

\begin{abstract}
Introduction: Time series models are one of the frequently used methods to describe the pattern of spreading an epidemic.

Methods: We presented a new family of time series models able to represent the cumulative number of individuals that contracted an infectious disease from the start to the end of the first wave of spreading. This family is flexible enough to model the propagation of almost all infectious diseases. After a general discussion on competent time series to model the outbreak of a communicable disease, we introduced the new family through one of its examples.

Results: We estimated the parameters of two samples of the novel family to model the spreading of COVID-19 in China.

Discussion: Our model does not work well when the decreasing trend of the rate of growth is absent because it is the main presumption of the model. In addition, since the information on the initial days is of the utmost importance for this model, one of the challenges about this model is modifying it to get qualified to model datasets that lack the information on the first days.
\end{abstract}

Keywords: family; GJR model; infection COVID-19; model; outbreak; spreading; time series.

\section{Introduction}

Recent years have seen a rising trend in the number of studies utilizing mathematical models. These studies are mainly based on seminal research including (Bartlett 1949; Diekmann et al. 1990; En'ko 1989; Hamer 1906; Kermack and McKendrick 1927; Ross 1911) that have introduced the models to increase understanding of what is happening, and to help us in terms of prediction and insight. A mathematical model uses mathematical concepts to give a more refined, detailed, and advanced description of the subject matter. In epidemiology, models allow us to predict the population-level epidemic dynamics from an individual-level knowledge of epidemiological factors, the long-term behavior from the early invasion dynamics, or the impact of vaccination on the spread of infection (Brauer, Castillo-Chavez, and Feng 2019). These models often identify behaviors that are unclear in experimental data, because the intended data are non-reproducible and the number of data points is limited and subject to errors in measurement (Brauer and Castillo-Chavez 2010).

Generally, formulating a model is accompanied by a trade-off between three important and often conflicting elements: accuracy, transparency, and flexibility. These three elements refer to the ability to reproduce the

\footnotetext{
*Corresponding author: Babak Jamshidi, Postdoctoral Researcher, Kermanshah University of Medical Sciences, Kermanshah, Iran, E-mail: babak.j6668@gmail.com

Shahriar Jamshidi Zargaran, Tehran University of Medical Sciences, Tehran, Iran, E-mail: shahriarjamshidy@gmail.com Mansour Rezaei, Kermanshah University of Medical Sciences, Kermanshah, Iran, E-mail: mrezaei@kums.ac.ir
} 
observed data and predict future dynamics reliably, being understandable analytically (how the components of the model interact and influence the dynamics), and adaptability to new or different situations, respectively (Brauer, Castillo-Chavez, and Feng 2019). It is clear that no model is perfect generally, and particularly in epidemiology, no model can exactly predict the detailed outcome of an infection process. However, a good model should be as simple as possible, but no simpler; A model designed to describe the behavior of an infectious disease should focus on the characteristics that are of interest while simplifying all others. Also, a suitable model should be parameterizable from available data (Brauer, Castillo-Chavez, and Feng 2019).

The methods applicable to model propagation of a disease are: Agent-based models (Smith et al. 2018), Network models (Firth et al. 2020; Jamshidi, Alavi, and Parham 2019), Stochastic and deterministic differential equation models (Okabe and Shudo 2020; Zhang et al. 2020), Compartmental models (Brauer 2008), Multi-state models (Ruiz-Castro and Zenga 2020), Time series models (Jamshidi, Rezaei, and Rezaei 2020a; Jamshidi et al. 2020b; Li et al. 2012; Nobre et al. 2001; Zhang et al. 2013; Zhang et al. 2014), Artificial intelligence models (Car et al. 2020; Wieczorek, Siłka, and Woźnia 2020), Growth function models (Musa et al. 2020; Wu et al. 2020), and combination of them.

The formulation of this family of time series is different from others. It is based on a recursive formula. Although the time series models have long been of interest in the literature, to the best of our knowledge, theses applied time series models including (Li et al. 2012; Nobre et al. 2001; Zhang et al. 2013; Zhang et al. 2014) are restricted to the ARIMA family. The present paper attempts to address this shortage as well.

Considering the graphs regarding seven outbreaks of communicable diseases in Plot 1, we understand that about all of them, the rate of growth follows a falling trend. This falling procedure is illustrated by partitioning the graph to three segments; at first, Exponential, Then, Linear, and Finally, Logarithmic (Plot 1). Accordingly, we can categorize the behavior of a plot of an infection on a population into three stages;

Stage 1. The initiation of the disease (step 1 in the formulation).

Stage 2. The beginning of the awareness, and therefore precaution and control measurements (step 2 in formulation).

Stage 3. The phase of the retreat of the disease leading to the end of the first wave of spreading (steps 3, 4, 5, and ... in the formulation).

It is worth saying that the stages are not equivalent to the partitions depicted by Plot 1 .

Considering all the points above, we want to define a new model based on the following assumptions:

A.1. During the first days, the infection faces no resistance or control and its rate of spreading is extreme (Britton et al. 2014). As time passes, the rate of growth decreases, and after a while, the rate of growth approaches one (The end of the first wave of spreading).

A.2. The model deals with the spreading of the disease since the first days.

To the best of our knowledge, Mathematical epidemic models mainly address Stage 1, and in the most advanced cases, deal with Stage 2 too. However, we present a novel model called Generalized Jamshidi-Rezaei (GJR) model that can represent whole of the procedure of the outbreak of the disease from stage 1 to stage 3 of all communicable diseases.

\section{Mathematical definition of the model}

At first, it is noticeable that a time series model as a model for the propagation of an infectious disease needs to possess the following parameters in order that be flexible enough to describe all the stages as simple as possible:

P1. The number of infected cases at the initial time (The models that lack this parameter let this equal to 1).

P2. The number of the first days (Most of the models just deal with the initial days of the outbreak, therefore, they do not need this parameter).

P3. The rate of growth over the first days. 


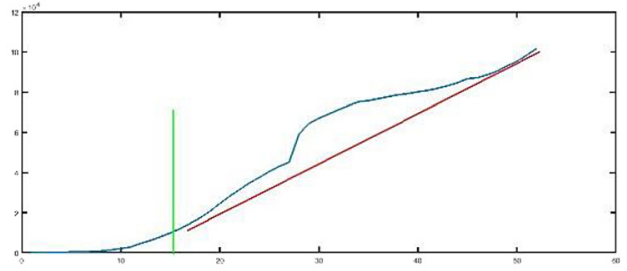

A

B
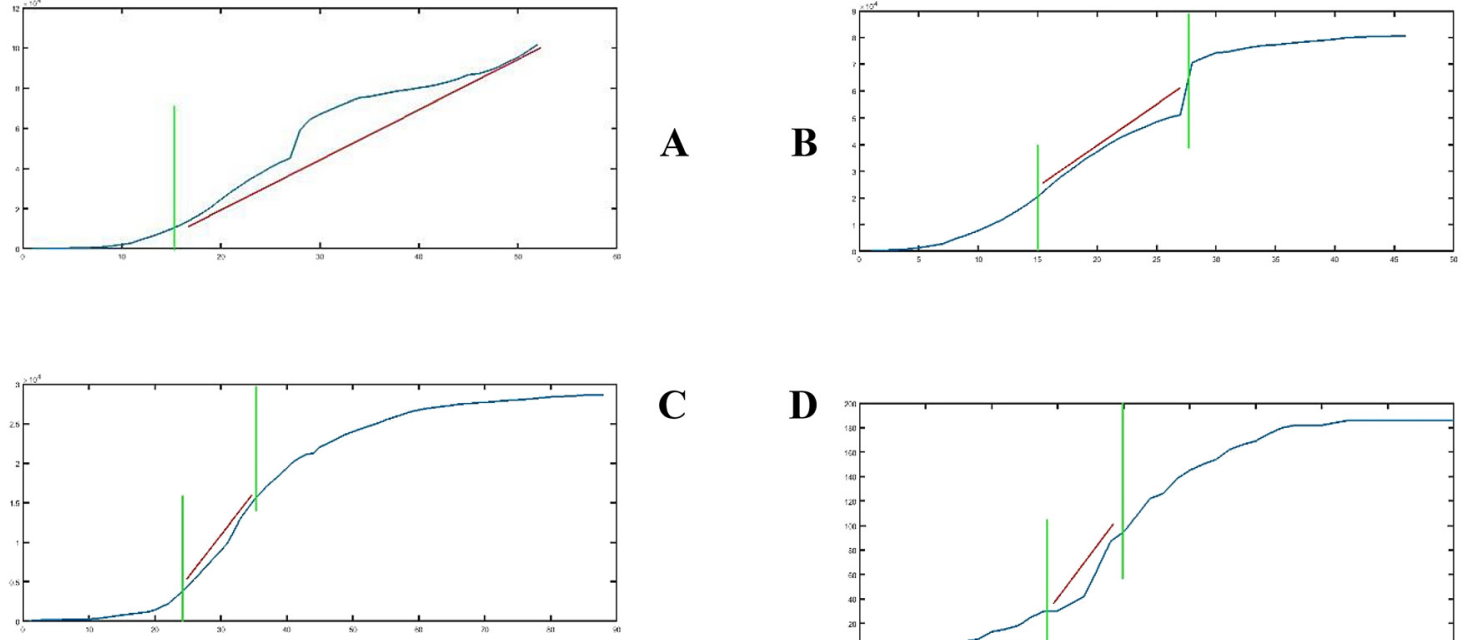

C
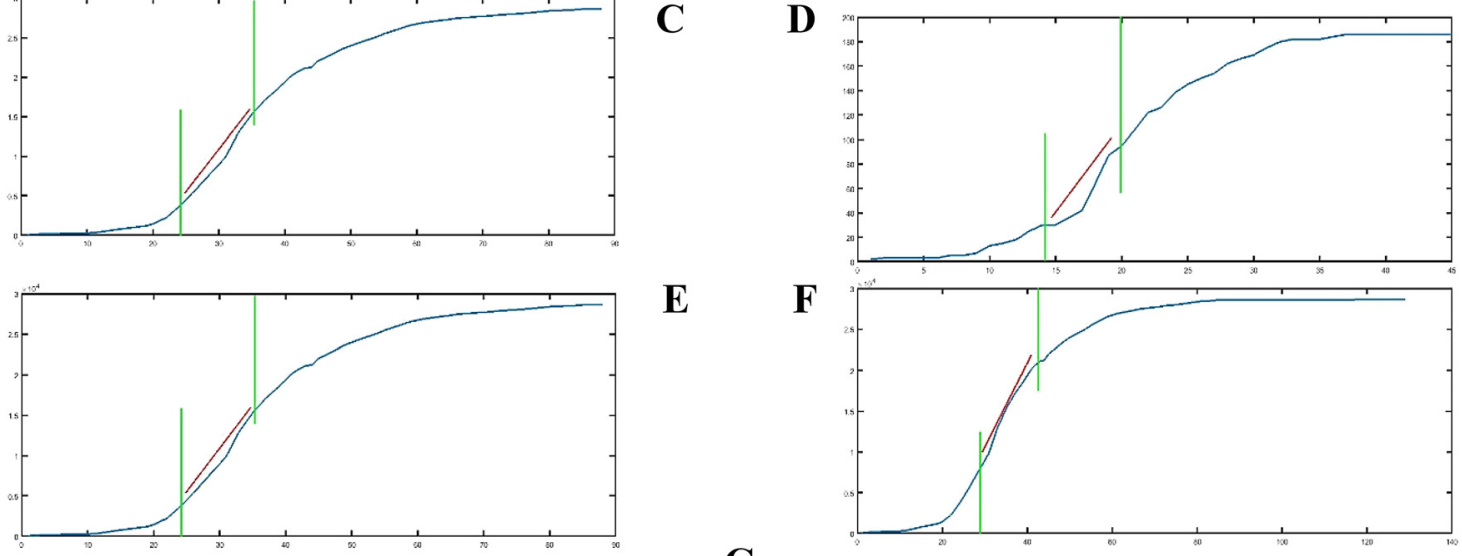

$\mathbf{E}$

$\mathbf{F}$

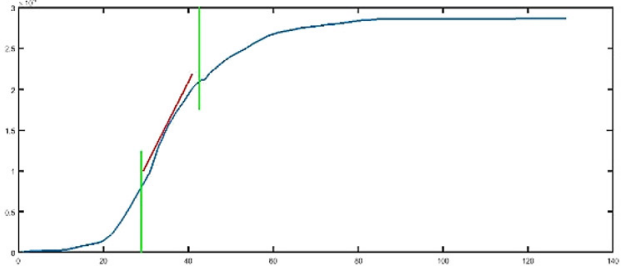

G

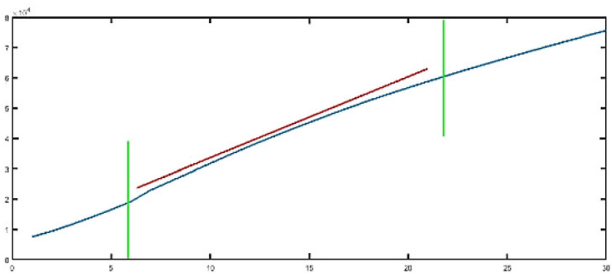

Plot 1: 1.A. The cumulative number of reported cases of COVID-19 worldwide (daily Jan 21st - Mar 7th , 2020), 1.B. COVID-19 in China (daily Jan 21st - Mar 7th , 2020), 1.C. SARS (daily Mar 17th - June 11th ,2003), 1.D. MERS in South Korea (daily May 20th - July 7th , 2018), 1.E. Ebola worldwide (weekly Mar 22nd ,2014 - June 10th, 2016), 1.F. Cholera in Zimbabwe (daily 11th Nov, 2008 - 9th Feb, 2009), and 1.G. HIV/AIDS estimated cumulative number of infected individuals (Annual 1990-2018) (UN 2019; WHO 2003; WHO 2008-9; WHO 2014-5; WHO 2018; WHO 2020).

Almost all the models, both graphical and non-graphical, both stochastic and deterministic, both complicated and simple, are based on the exponential growth $\left(e^{\lambda t}\right)$ in the initial days $(t=1,2, \ldots, b)$.

P4. At least one parameter to model the falling rate of growth Usually, it is assumed that the rate decreases continuously or at some specified times like the start of control measurements or discovery of the treatments. Also, it is possible to define a mechanism to cover the gradual and steep decreases in trend.

P5. A randomizing factor to promote the competency and flexibility of the model

It is worth saying that we can add a part similar to noise component dependent on at least one parameter to model the partial violations of the model.

As an example of our family, we present a model to cover all the procedure of the disease; $X_{t}=G J R(x, b, R, l$, a) with five parameters;

$x=$ The number of infected cases at the initial time (P1)

$b=$ The number of the first days (P2) 
$R=$ The rate of the growth in the initial days $(R=\exp (\lambda))(P 3)$

$l=$ The number of consecutive $O$ s of the variable $U$ that increases the step of the process by one (steep falling)

(P4)

$p=$ The parameter related to the decreasing probability of the rate of the spreading corresponding to different items in a specified step (gradual falling) (P4)

Our model is formulated as:

I. The initial condition

$$
\begin{gathered}
\text { Step }_{1}=\text { Step }_{2}=\cdots=\text { Step }_{b}=1 \text { and } \text { Step }_{b+1}=2 \\
\text { Item }_{1}=1 \\
U_{1}=U_{2}=\cdots=U_{(b-1)}=0 \\
X_{1}=x
\end{gathered}
$$

II. The evolution law

$$
\begin{aligned}
& \text { Step }_{(t+1)}= \\
& \text { Step }_{t}+\prod_{j=1}^{l} U(t-j)\left(1-\text { Step }_{t-j}+\text { Step }_{t-j-1}\right) \quad t=b+1, b+2, \ldots \\
& \operatorname{Item}_{(t+1)}=1+\operatorname{Item}_{t}\left(1-\text { Step }_{t+1}+\text { Step }_{t}\right) \quad t=b, b+1, \ldots \\
& U_{(t+1)} \sim \operatorname{Bernouli}\left(p^{\text {Item }_{(t+1)}}\right) \quad t=b, b+1, b+2, \ldots \\
& X_{t+1}=X_{t}\left(1-\frac{1}{R^{\operatorname{Step}_{(t+1)}+U_{(t+1)}-1}}+\frac{1}{R^{\operatorname{Step}_{(t+1)}+U_{(t+1)}-2}}\right) \quad t=2,3,4, \ldots .
\end{aligned}
$$

Therefore, we can classify this model as a multivariate time series wherein,

$X_{t}$ : The cumulative number of infected individuals at time $t$

Step $_{t}$ : The phase of the outbreak of the disease at time $t(\mathrm{P} 4)$

Item $_{t}$ : The item of the disease at time $t(\mathrm{P} 4)$

It is noticeable that the variable Item is more detailed and more partial in comparison with the variable Step. Also, the introduced rate of growth is equal to daily relative increment plus 1.

$U_{t}$ : The intensity of spreading at time $t$ (dependent on the variable Item) (P5)

The rationale behind the model is that the proportion of the transmitting cases among infected cases is decreasing. In the beginning, this proportion is equal to one. As time goes by, it falls to $1 / R^{1}, R^{2}, \ldots, 1 / R^{n}, \ldots$, respectively. The random variable $U$ connects these levels continuously and makes the process seem continuous. Therefore, in the above model, we assume that the probability of acute outbreaks in each step decreases exponentially and conversely, the probability of mild and gradual increments increases. In addition, we assume that the rate of growth is falling gradually, and it decreases significantly if a run of weak growths happens.

Using this model, we are able to compare the propagation of a disease in some populations $(\mathrm{A}, \mathrm{B}, \ldots)$ much better, since we have two factors to compare; $R$ and Step. It is possible that despite the less $R$ in comparison with $B$, population $A$ have an upper growth of the infection than $B$ because of the less stage of the outbreak in it.

Here, we present some notes to take into consideration for generalizing the model and constructing the GJR model:

(1) The length of the run can be constant, decreasing, or increasing dependent on the steps of the process. Accordingly, the model is called Fixed GJR, Decreasing GJR, or Increasing GJR, respectively.

(2) The mechanism of the decreasing probability of the more intense changes can be modeled by any decreasing function such as exponential $\left(p^{\text {Item }}\right)$, logarithmic $(p / \log ($ Item $+c)$, fractional $(p /$ Item $)$, and generalized fractional $\left(p /(\text { Item })^{\alpha}\right)$. Accordingly, the model is called Exponential GJR, Logarithmic GJR, Fractional GJR, or Generalized fractional GJR, respectively. 
(3) The randomizer distribution can differ from Bernoulli distribution, and it can take more than two values. It can take negative values to reduce Items and Steps as well.

(4) It is possible to adopt a Fuzzy approach to the model and its parameters to increase its flexibility.

It is well known that there are some models introduced to describe specifically the time series of the spreading of the diseases Tuberculosis, HIV/AIDS, Influenza, Ebola, Malaria, Zika, ... See (Brauer, Castillo-Chavez, and Feng 2019) for examples of each of them. However, our novel family presents some models suitable for all communicable diseases.

Finally, we apply two models of this family to replica the pattern of the cumulative number of confirmed cases infected by COVID-19 in China from Jan 21st to March 7th; Fixed Exponential GJR and Fixed Fractional GJR. To estimate the parameters of these two models, we apply the method of (Jamshidi, Rezaei, and Rezaei 2020a) as an example of the models belonging to this family. The calculations lead to parameters $(278,1.3595$, $13,2,0.0408)$ and $(278,1.3595,13,2,0.039)$ for the models Fixed Exponential GJR and Fixed Fractional GJR, respectively. Plot 2 presents two couple of the mean curves obtained by averaging 100 realizations of the two mentioned fitted models. According to Plot 2, it is obvious that the models fit the process remarkably well.

\section{Our model vs. the logistic growth function}

A logistic function or logistic curve is a common S-shaped curve (sigmoid curve) with equation

$$
f(x)=\frac{L}{1+\exp \left(-k\left(x-x_{0}\right)\right)} f
$$

where

$x_{0}=$ the $x$ value of the midpoint,

$L L=$ the curve's maximum value, and

$k=$ the logistic growth rate or steepness of the curve (Verhulst 1838).

The presumptions of this model are:

- The carrying capacity of a particular environment is the maximum population size that it can support.

- The carrying capacity acts as a moderating force in the growth rate by slowing it when resources become limited and stopping growth once it has been reached.

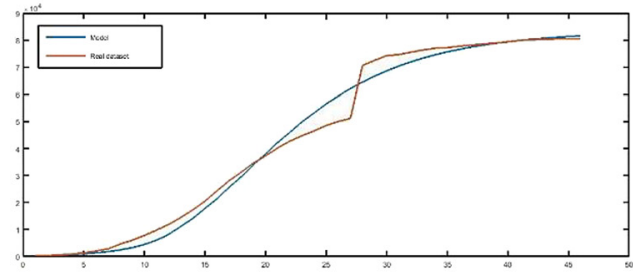

A
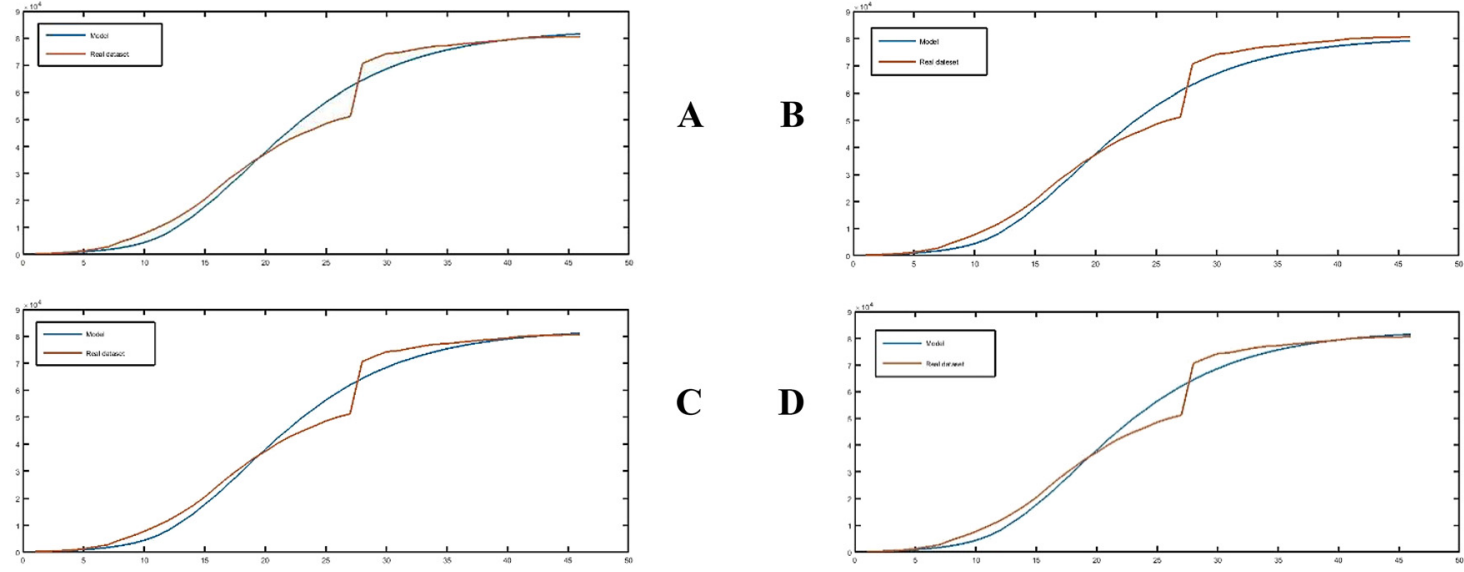

C

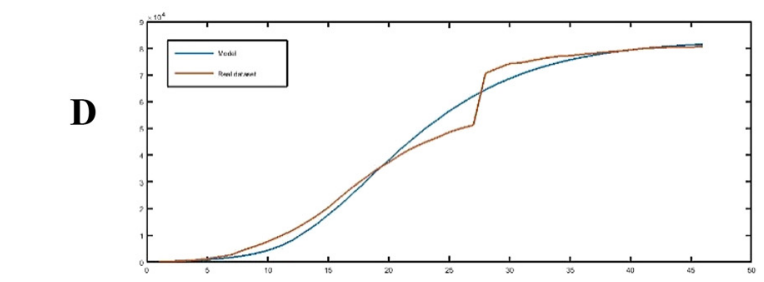

Plot 2: Two mean curves of 100 simulations of fixed exponential $G J R(278,1.3595,13,2,0.0408)$ (2.A. and 2. B.) and two mean curves of 100 simulations of fixed fractional GJR $(278,0.039,13,2,1.3595)$ (2.C. and 2. D.) for Jan 21st - March 7 th. 
- As population size increases and resources become more limited, intraspecific competition occurs: individuals within a population who are more or less better adapted for the environment compete for survival.

This model is symmetric and this is a simplifier assumption that is usually not met in real-world situations. Our model is a time series model to describe the daily relative increment, while Logistic growth model is a function (at most by adding an error term is a regression) to describe the number of recovered individuals in an SIR model. Trivially, both of these models are suitable for representing similar curves as well. The Logistic growth function is a 3-parameter model while our model is a 5-parameter model. Due to these two additional parameters, our model is much more flexible (Figure 1 (Jamshidi, Rezaei, and Rezaei 2020a)) than Logistic growth model. It is remarkable that the restricted form of our model (Jamshidi, Rezaei, and Rezaei 2020a) is able to describe the following situations:

- Modelling the sequence of Facebook users from 2014 to 2019,

- Modelling the time series of confirmed cases of SARS in China from March 17 to June 17, 2003, and

- Modelling the procedure of COVID-19 from January 13 to March 5, 2020.

\section{Discussion and conclusion}

We presented a novel family of time series models to deal with the cumulative number of individuals that contracted a disease from the start to the end of the first wave of spreading.

This family of the models allows us to provide analysis for the spreading of infection worldwide while other models are applicable for regional analysis at most. However, considering it locally or by country is more accurate in comparison with worldwide. One of the merits of this model is the capability of being updated. Regarding the prediction of the infections like COVID-19 worldwide, the emerging new epicenters predicted can be modeled through decreasing the step of the process by 1 unit at times predicted. On the other hand, awareness is modeled through the increased step from 1 to 2 . Intensifying the controls and warnings causes the infection to spread at a lower rate, and this falling rate is modeled through the rising steps in our model. It is noticeable that the effect of the discovery of a treatment or making a vaccine can be modeled in a similar way.

Like other time series, the models of this family require at least a number of data to estimate their parameters. In this case, because of the existence of five parameters and the need for at least three steps of the process, this requirement is more essential. Besides, the more information we have, the booster model and the more accurate estimations we can obtain.

Our model does not work well when the decreasing trend of the rate of growth is absent because it is the main presumption of the model. In addition, since the information on the initial days is of the utmost importance for this model, one of the challenges about this model is modifying it to get qualified to model datasets that lack the information on the first days. Finally, we can define a new family of models; the logarithm of this family to model the logarithm of the cumulative number of infected individuals. One of the advantages of these logarithmic models is that they deal with the digits much less. Therefore, they can be more accurate in comparison with the original models introduced in the present paper.

Research funding: None declared.

Author contributions: All authors have accepted responsibility for the entire content of this manuscript and approved its submission.

Competing interests: Authors state no conflict of interest.

\section{References}

Bartlett, M. 1949. “Some Evolutionary Stochastic Processes." Journal of the Royal Statistical Society. Series B (Methodological) 11 (2): 211-29. 
Brauer, F. 2008. “Compartmental Models in Epidemiology.” In Mathematical Epidemiology. Lecture Notes in Mathematics, 1945, edited by F. Brauer, P. van den Driessche, and J. Wu Berlin, Heidelberg: Springer. https://doi.org/10.1007/978-3-540-78911-6_2. Brauer, F., and C. Castillo-Chavez. 2010. Mathematical Models in Population Biology and Epidemiology, 2nd ed. Newyork: Springer. Brauer, F., C. Castillo-Chavez, and Z. Feng. 2019. Mathematical Models in Epidemiology. Newyork: Springer.

Britton, T., T. House, A. L. Lloyd, D. Mollison, S. Riley, and P. Trapman. 2014. "Five Challenges for Stochastic Epidemic Models Involving Global Transmission." Epidemics 10: 54-7.

Car, Z., S. Baressi Šegota, N. Anđelić, I. Lorencin, and V. Mrzljak. 2020. Modeling the Spread of COVID-19 Infection Using a Multilayer Perceptron, Computational and Mathematical Methods in Medicine. Article ID 5714714, https://doi.org/10.1155/ 2020/5714714.

Diekmann, O., J. Heesterbeek, and J. A. Metz. 1990. "On the Definition and the Computation of the Basic Reproduction Ratio R0 in Models for Infectious Diseases in Heterogeneous Populations.” Journal of Mathematical Biology 28 (4): 365-82.

En'ko, P. D. 1989. “On the Course of Epidemics of Some Infectious Diseases.” International Journal of Epidemiology 18 (4): 749-55. Firth, J. A., J. Hellewell, P. Klepac, S. Kissler, A. J. Kucharski, and L. G Spurgin. 2020. “Using a Real-World Network to Model Localized COVID-19 Control Strategies." Nature Medicine 26: 1616-22.

Hamer, W. H. 1906. The Milroy Lectures on Epidemic Disease in England: The Evidence of Variability and of Persistency of Type. London: Bedford Press.

Jamshidi, B., S. M. R. Alavi, and G. A. Parham. 2019. "The Distribution of the Number of the Infected Individuals in a Stochastic SIR Model on Regular Rooted Trees." Communications in Statistics - Simulation and Computation:1-18, https://doi.org/10.1080/ 03610918.2019.1584299.

Jamshidi, B., M. Rezaei, and K. Rezaei. 2020a. “A New Model for Epidemic Spreading with a Focus on COVID-19.” Health Scope 9 (3). Jamshidi, B., H. Bekrizadeh, S. Jamshidi Zargaran, and M. Rezaei. 2020b. “A New Family of Time Series to Model the Decreasing Relative Increment of Spreading of an Outbreak (Focused on COVID-19 in China).” Preprint.

Kermack, M., and A. G. McKendrick. 1927. “Contributions to the Mathematical Theory of Epidemics." Part I, Proceedings of the Royal Society. Series A, Containing Papers of a Mathematical and Physical Character 115 (5): 700-21.

Li, Q., N. N. Guo, Z. Y. Han, Y. B. Zhang, S. X. Qi, Y. G. Xu, Y. M. Wei, X. Han X, and Y. Y. Liu. 2012. "Application of an Autoregressive Integrated Moving Average Model for Predicting the Incidence of Hemorrhagic Fever with Renal Syndrome." The American Journal of Tropical Medicine and Hygiene 87: 364-70.

Musa, S. S., S. Zhao, M. H. Wang, A. G. Habib, and U. T. Mustapha. 2020. "Estimation of Exponential Growth Rate and Basic Reproduction Number of the Coronavirus Disease 2019 (COVID-19) in Africa." Infectious Diseases Poverty 9: 96.

Nobre, F. F., A. B. S. Monteiro, P. R. Telles, and G. D. Williamson. 2001. "Dynamic Linear Model and SARIMA: A Comparison of Their Forecasting Performance in Epidemiology.” Statistics in Medicine 20: 3051-69.

Okabe, Y., and A. Shudo. 2020. “A Mathematical Model of Epidemics-A Tutorial for Students.” Mathematics 8 (7): 1174.

Ross, R. 1911. "Some Quantitative Studies in Epidemiology." Nature 87: 466-7.

Ruiz-Castro, J. E., and M. Zenga. 2020. "A General Piecewise Multi-State Survival Model: Application to Breast Cancer.” Statistical Methods and Applications 29: 813-43.

Smith, N. R., J. M. Trauer, M. Gambhir, J. S. Richards, R. J. Maude, J. M. Keith, and J. A. Flegg. 2018. "Agent-based Models of Malaria Transmission: a Systematic Review." Malaria Journal 17: 299.

UN. 2019. Global HIV and AIDS Statistics. Also available at https://www.unaids.org/en/resources/fact-sheet.

Verhulst, P. F. 1838. “Notice Sur La Loi Que La Population Poursuit Dans Son Accroissement.” Correspondance Mathématique et Physique 10: 113-21 [Retrieved December 3 2014].

WHO. 2003. Severe Acute Respiratory Syndrome (SARS) Situation Reports: March - July 2003. Also available at https://www.who. int/csr/sars/country/en/.

WHO. 2008-9. Cholera Situation Reports. Also available at https://www.who.int/csr/don/archive/country/zwe/en/.

WHO. 2014-5. Ebola Situation Reports: March 2014 - November 2015. Also available at https://www.who.int/csr/disease/ebola/ situation-reports/archive/en/.

WHO. 2018. MERS-cov Situation Reports: May - July 2018. Also available at https://www.who.int/csr/don/archive/disease/ coronavirus_infections/en/.

WHO. 2020. Coronavirus Disease 2019 (COVID-19) Situation Reports: 1 to 44. January, February, and March 2020. Also available at https://www.who.int/docs/default-source/coronaviruse/situation-reports/.

Wieczorek, M., J. Siłka, and M. Woźniak. 2020. “Neural Network Powered COVID-19 Spread Forecasting Model, Chaos.” Solitons \& Fractals 140, https://doi.org/10.1016/j.chaos.2020.110203.

Wu, K., D. Darcet, Q. Wang, and D. Sornette. 2020. "Generalized Logistic Growth Modeling of the COVID-19 Outbreak: Comparing the Dynamics in the 29 Provinces in China and in the Rest of the World." Nonlinear Dynamics 101: 1561-81.

Zhang, X., Y. Liu, M. Yang, T. Zhang, A. A. Young, and X. Li. 2013. "Comparative Study of Four Time Series Methods in Forecasting Typhoid Fever Incidence in China." PloS One 8: e63116.

Zhang, X., T. Zhang, A. A. Young, and X. Li. 2014. "Applications and Comparisons of Four Time Series Models in Epidemiological Surveillance Data.” PloS One 9 (2): e88075.

Zhang, Z., A. Zeb, S. Hussain, and E. Alzahrani. 2020. “Dynamics of COVID-19 Mathematical Model with Stochastic Perturbation.” Advances in Difference Equations 451, https://doi.org/10.1186/s13662-020-02909-1. 